\title{
Über analytische Operatorfunktionen und Indexberechnung
}

von

BERNHARD GRAMSCH (Mainz)

Sei $\mathscr{L}(X, Y)$ die Menge der stetigen linearen Abbildungen des Banachraumes $X$ in den Banachraum $Y$. Für eine Abbildung $T \in \mathscr{L}(X, Y)$. bezeichnen wir mit $N(T)$ den Nullraum und mit $R(T)$ den Bildraum. $T$ heißt Fredholmoperator, wenn $\operatorname{dim} N(T)<\infty$ und $\operatorname{codim} R(T)<\infty$. $\varphi(X, Y)$ sei die Menge der Fredholmoperatoren aus $\mathscr{Z}(X, Y)$.

1. THEOREM. Sei $T(z)$ eine analytische Operatorfunktion auf dem Gebiet $G \subset \boldsymbol{C}^{N}$ mit Werten in der Menge der Fredholmoperatoren $\varphi(X, Y)$. Ferner sei $T(z)$ an einer Stelle $z^{\prime} \in G$ invertierbar. Dann ist $T^{-1}(z)$ eine auf $G$ meromorphe Funtion $\left(T(z) T^{-1}(z)=I_{Y}, T^{-1}(z) T(z)=I_{X}\right)$. Im Falle einer komplexen Variablen hat $T^{-1}(z)$ als Hauptteile Operatoren von endlichem. Rang; d. $h$. für jedes $z_{0} \in G$ existiert eine Laurententwicklung

$$
T^{-1}(z)=\sum_{k>k_{0}\left(z_{0}\right)>-\infty} T_{k}\left(z-z_{0}\right)^{k}
$$

wobei $\operatorname{dim} R\left(T_{k}\right)<\infty$ für $k<0$.

Die ersten Untersuchungen in dieser Richtung gehen auf Tamarkin [10] für den Fall von Integralgleichungen zurück. Daran schließen sich Arbeiten von Atkinson [1] Gohberg und Sz. Nagy und P. H. Müller an. (vgl. [6] und [8]). Der klassische Satz von F. Riesz, daß $(I-z K)^{-1}$ für einen kompakten Operator $K$ eine auf der komplexen Ebene im obigen. Sinne meromorphe Funktion ist, wurde in einer Reihe von Arbeiten von. Ruston, Pietsch und Taylor bei linearer Abhängigkeit $(T(z)=I-z A$ $\epsilon \varphi(X, X)$ auf einem Gebiet $G)$ verallgemeinert. Im letzten Jahr wurden von Haf, Steinberg, Ribaric und Vidar [8] und dem Verfasser [4] weitere Fortschritte erzielt. Mit den Methoden von [4] und einer Idee in [8]: gelang es dann Theorem $1 \mathrm{zu}$ beweisen (vgl. [5]).

Theorem 1 läßt sich auf analytische Familien vom Typ A (Kato. [7], ch. 7, p. 379) anwenden. Dies sind abgeschlossene Operatoren, die- 
analytisch von einem Parameter abhängen und einen gemeinsamen Definitionsbereich haben. Insbesondere bezieht sich Theorem 1 auf elliptische Differentialoperatoren, die analytisch von Parametern abhängen, denn bei geeigneter Randbedingung handelt es sich dabei um Fredholmoperatoren.

Wir sagen ein Operator $A \in \mathscr{Z}(X)=\mathscr{Z}(X, X)$ erfüllt beide Kettenbedingungen, wenn sowohl die Nulliraumkette $N\left(A^{j}\right)$ als auch die Bildraumkette $R\left(A^{j}\right)$ stabil werden. Mit $\varphi^{R}$. (Fredholm-Riesz) bezeichnen wir die Menge der Fredholmoperatoren aus $\mathscr{Z}(X)$ mit beiden Kettenbedingungen.

2. THEOREM. Sei $T(z)$ eine kommutative $\left(T\left(z_{1}\right) T\left(z_{2}\right)=T\left(z_{2}\right) T\left(z_{2}\right)\right)$ analytische Operatorfunktion auf dem Gebiet $G \subset C^{N}$, die Werte in der Menge $\varphi(X)$ der Fredholmoperatoren von $\mathscr{Z}(X)$ habe; ferner existiere $T^{-1}\left(z^{\prime}\right)$ für ein $z^{\prime} \in G$. Dann gilt $T(z) \in \varphi^{R}$. für alle $z \in G$.

Es ist sehr einfach, die Meromorphie von $T^{-1}(z)$ aus Theorem 2 herzuleiten. Ohne die Annahme der Kommutativität ist Theorem 2 im allgemeinen falsch.

Um Theorem $2 \mathrm{zu}$ beweisen, benützt man die Tatsache, daß ein Operator $T \epsilon \mathscr{L}(X)$ genau dann in $\varphi^{R}$-liegt, wenn ein $A \in \mathscr{Z}(X)$ existiert mit $A T=T A=I-K$, wobei $K$ ein kompakter Operator ist. Daraus folgt unmittelbar

3. Lemma. Sei $\mathscr{M}$ eine maximale Kommutative Teilalgebra der Banachalgebra $\mathscr{L}(X)$ und $\mathscr{K}$ das abgeschlossene zweiseitige Ideal der kompakten Operatoren; ferner sei $q_{\mathscr{H}}: \mathscr{M} \rightarrow \mathscr{M} \mid \mathscr{M} \cap \mathscr{K}$ der kanonische Homomorphismus. Dann gilt

$$
\varphi^{R} \cap \mathscr{M}=q_{\mathscr{M}}^{-1}(\mathscr{G}(\mathscr{M} \mid \mathscr{M} \cap \mathscr{K})),
$$

wenn $\mathscr{G}(\mathscr{M} / \mathscr{M} \cap \mathscr{K})$ die Gruppe der invertierbaren Elemente der Banachalgebra $\mathscr{M} \mid \mathscr{M} \cap \mathscr{K}$ ist.

Dies entspricht der Charakterisierung von Atkinson für Fredholmoperatoren. Daraus gewinnt man sofort einen Spektralabbildungssatz. Für $\sigma_{R}(T)=\left\{\lambda \epsilon \sigma(T): \lambda I-T \notin \varphi^{R}\right\}(\sigma(T)$ sei das Spektrum von $T)$ gilt $\sigma_{R}(f(T))$ $=f\left(\sigma_{R}(T)\right)$, wenn $f$ eine auf dem Spektrum von $T$ lokalholomorphe Funktion ist. Eine entsprechende Aussage gewinnt man ebenso für lokalholomorphe Funktionen $f$ auf dem gemeinsamen Spektrum $\sigma\left(T_{1}, \ldots, T_{n}\right)$ kommutierender Operatoren $T_{j}: f\left(T_{1}, \ldots, T_{n}\right)$ ist genau dann ein Element von $\varphi^{R}$, wenn $f$ auf $\sigma\left(q_{\mathscr{M}}(T), \ldots, q_{\mathscr{M}}(T)\right)$ keine Nullstelle hat (vgl. [2] und [3]).

4. Bemerkung. Die Menge $\mathscr{M}(G, \varphi)$ der auf einem Gebiet $G \subset C$ meromorphen Funktionen mit Werten in $\varphi(x)$, die jeweils an einer Stelle von $G$ invertierbar sind und lokale Laurententwioklungen wie in Theorem 1 haben $\left(T_{0} \epsilon \varphi\right)$, bilden eine multiplikative Gruppe, d. h. die Inversenbildung führt aus dieser Klasse nicht heraus.

Wir gehen nun zur Berechnung des Index von Fredholmoperatoren über: ind $T=\operatorname{dim} N(T)-\operatorname{codim} R(T)$.

5. BEMERKUNG. Fï̈r Fredholmoperatoren auf Hilberträumen gilt

$$
\begin{aligned}
\operatorname{ind} T & =\operatorname{Spur}\left[\lim \lambda_{\lambda \rightarrow 0}\left(\left(\lambda I-T^{*} T\right)^{-1}-\left(\lambda I-T T^{*}\right)^{-1}\right)\right] \\
& =\operatorname{Spur}\left[\frac{1}{2 \pi i} \int_{\gamma}\left(\left(\lambda I-T^{*} T\right)^{-1}-\left(\lambda I-T T^{*}\right)^{-1}\right) d \lambda\right],
\end{aligned}
$$

wobei der Grenzwert $\lambda \rightarrow 0$ in der Normtopologie von $\mathscr{Z}(H)$ existiert und $\gamma$ ein genügend kleiner Kreis um den Nullpunkt ist.

(Die Spur eines Operators $A$ der Spurklasse ist $\sum_{k}\left(A e_{k_{k}}, e_{k}\right)$ für ein Follständiges Orthonormalsystem $\left\{e_{k}\right\}$ des Hibertraumes $H$.)

Wir untersuchen nun den Index von Fredholmoperatoren in Verbindung mit einem Funktionalkalkül $\psi$, das heißt $\psi$ sei ein Homomorphismus einer Funktionenalgebra $\mathscr{F}(\Omega)$ in $\mathscr{Z}(X)$ bzw. in eine Banachalgebra $\mathscr{Z}$ mit $\psi(1)=I$. Man betrachte z.B. den analytischen Funktionalkalkül von Gelfand und Dunford oder den Kalkül von Schilow-Arens-Waelbroeck (vgl. [2]). Der Index von Fredholmoperatoren ist invariant modulo dem Ideal $\mathscr{K}$ der kompakten Operatoren von $\mathscr{Z}(X)$; nach der Charakterisierung von Atkinson kann man ihn deshalb über die Gruppe $T$ der invertierbaren Elemente von $\mathscr{Z} / \mathscr{K}$ faktorisieren: ind $=q \cdot \hat{\mathfrak{i}}$, dabei ist $q: \mathscr{Z} \rightarrow \mathscr{Z} / \mathscr{K}$ der kanonische Homomorphismus und $\hat{\mathfrak{t}}: \Gamma \rightarrow 3$ der vom Index induzierte Homomorphismus in die ganzen Zahlen.

6. BEMERKUNG. Sei i: $T \rightarrow 3$ ein Homomorphismus der Gruppe $\Gamma$ der invertierbăren Elemente einer Banachalgebra $\mathscr{B}$ in (auf) eine Gruppe 3, wobei für kein vom Einselement in 3 verschiedenes Element a zu jeder natürlichen Zahl $n$ eine n-te Wurzel a existiert, d.h. $a_{n}^{n}=a$ bzw. für eine abelsche Gruppe $3 n a=a$ (freie abelsche Gruppen haben diese Eigenschaft). Dann ist der Homomorphismus $\mathrm{i}$ auf jeder Zusammenhangskomponente von $\Gamma$ konstant.

Beweis. Es genügt zu zeigen, daß $\mathfrak{i}(x)$ auf einer Umgebung des Einselementes von $\Gamma$ gleich dem Einselement von 3 ist. Für $\|x-e\|<1$ existiert ein $y \in \mathscr{B}$ mit $x=\exp y$, folglich wäre $\left(\mathfrak{i}(\exp (y / n))^{n}=\mathfrak{i}(x)\right.$ für jede natürliche Zahl $n$, womit sich ergibt, daß $\mathfrak{i}(x)$ das Einselement von 3 sein muß.

Da man den üblichen Index für Fredholmoperatoren über die Gruppe $\Gamma$ der Restklassenalgebra $3 / \mathscr{K}$ faktorisieren kann, setzen wir im folgenden voraus, daß $\mathfrak{i}$ ein lokalkonstanter Homomophismus der. Gruppe $\Gamma$ der 
invertierbaren Elemente einer Banachalgebra $\mathscr{B}$ mit Einselement $e$ in (auf) eine Gruppe 3 ist. Ferner sei $\mathscr{F}(\Omega)$ eine Banachalgebra stetiger Funktionen auf dem kompakten Raum $\Omega$ (nicht notwendig Sup-NormAlgebra) und $\psi: \mathscr{F}(\Omega) \rightarrow \mathscr{B}$ ein stetiger Homomorphismus mit $\psi(1)=e$; außerdem sei $\mathscr{G}(\mathscr{F})$ die Gruppe der invertierbaren Elemente von $\mathscr{F}(\Omega)$. Wir erhalten dann eine Folge von Homomorphismen $(\mathfrak{i}=\hat{\mathfrak{i}} \cdot \pi)$

$$
\mathscr{G}(\mathscr{F}) \stackrel{\leftrightarrow}{\rightarrow} \Gamma \stackrel{\pi}{\rightarrow} \pi_{0}(\Gamma) \stackrel{\hat{i}}{\rightarrow} 3,
$$

wobei $\pi_{0}(\Gamma)$ die Gruppe der Zusammenhangskomponenten von $\Gamma$ ist.

Mit Hilfe eines tiefliegenden Ergebnisses von Arens und Royden ([9], S. 290-295) ergibt sich eine Möglichkeit, den Index $i(\psi(f))$ für $f \in \mathscr{G}(\mathscr{F}) \mathrm{zu}$ berechnen.

7. LeMMa. Ist $f \in \mathscr{G}(\mathscr{F})$ und $\log f \epsilon \mathscr{F}(\Omega)$ (d.h. insbesondere eindeutig), dann gilt $i(\psi(f))=e_{3}(=0$ falls 3 eine additive Gruppe).

Die Idee des folgenden Ergebnisses geht auf eine einfache Formel zur Indexberechnung in [2], $\$ 8$, zurück.

8. THEOREM. Fü̈r den Raum $\Lambda$ der maximalen Ideale von $\mathscr{F}(\Omega)$ gelte $\Lambda=\Omega$. Ferner seien $a_{1}, \ldots, a_{n}$ kommutierende Elemente der Banachalgebra $\mathscr{B}$ mit dem gemeinsamen Spektrum $\sigma\left(a_{1}, \ldots, a_{n}\right)$, das in der rational konvexen. Menge $\Omega \subset C^{n}$ (vgl. [2], S. 336) enthalten sei. Wenn $\psi: \mathscr{F}(\Omega) \rightarrow \mathscr{B}$ eine Fortsetzung des Kalkiuls von Waelbroeck (vgl. [2]) ist, d.h. für die Koordinatenfunktionen $z_{j}$ gilt $\psi\left(z_{j}\right)=a_{j}$, dann gibt es zu jeder Funktion $f \in \mathscr{G}(\mathscr{F})$, endlich viele Primpolynome $p_{k}$ und ganze Zahlen $\alpha_{k}$, so daß

erfüllt ist; bzw.

$$
\mathfrak{t}(\psi(f))=\prod_{k} \mathfrak{i}\left(p_{k}\left(a_{1}, \ldots, a_{n}\right)\right)^{a_{k}}
$$

$$
\mathfrak{i}(\psi(f))=\sum_{k} \alpha_{k} i\left(p_{k}\left(a_{1}, \ldots, a_{n}\right)\right)
$$

falls 3 eine additive Gruppe ist.

Durch eine geeignete Anwendung des Satzes von Arens und Royden [2], S. 290-295, ergibt sich, daß in jeder Zusammenhangskomponente von $\mathscr{G}(\mathscr{F})$ wenigstens eine rationale Funktion liegt, auf die man dann Primfaktorzerlegung anwenden kann.

Da jede kompakte Teilmenge der komplexen Ebene rational konvex ist und für $n=1$ die Primpolynome über $C$ die Gestalt $(z-c)$ haben, läßt sich in diesem Fall eine sehr einfache Formel angeben. Mit $\varrho(a)$ bezeichnen wir das Komplement des Spektrums $\sigma(a)$. Sei

$$
\left.\varrho_{h}(a)=\{\lambda \in \varrho(a): i(\lambda e-a)=h \epsilon\}\right\}
$$

dann gilt für eine rationale Funktion $r \in \mathscr{G}(\mathscr{F})$

$$
i(\psi(r))=I h^{\alpha_{h}}\left(=\sum \alpha_{h} h\right)
$$

wenn $\alpha_{h}$ die Anzahl der Nullstellen bzw. Pole von $r$ in $\varrho_{h}(a)$ ist. Dies folgt unmittelbar aus

$$
r=\frac{\prod\left(z-u_{i}\right)^{\beta_{j}}}{\prod\left(z-v_{k}\right)^{\gamma_{k}}}
$$

wenn man bei Anwendung von $\psi$ die Variable $z$ durch $a$ ersetzt (vgl. [2], §8).

\section{Literaturnachweis}

[1] F. V. Atkinson, A spectral problem for completely continuous operators, Acta Math. Hung. 3 (1952), S. $53-60$.

[2] B. Gramsch, Funktionalkalkül mehrerer Veränderlichen in lokalbeschränkten Algebren, Math. Ann. 174 (1967), S. 311-344.

[3] - Speltraleigenschaften analytischer Operatorfunktionen, Math. Zeitschrift 101 (1967), s. 165-181.

[4] - Über analytische Störungen und den Index von Fredholmoperatoren auf Banachräumen, Dept. Math., Univ. of Maryland TR 69/105 (1969), S. 1-55.

[5] - Meromorphie in der Theorie der Fredholmoperatoren mit Anwendungen auf elliptische Differentialoperatoren Math. Annalen 188 (1970), S. 97-112.

[6] S. Hildebrandt, Über die Lösung nichtlinearer Eigenwertaufgaben mit dem Galerkinverfahren, Math. Zeitschrift 101 (1967), S. 255-264.

[7] T. Kato, Perturbation theory for linear operators, Heidelberg 1966.

[8] M. Ribaric, I. Vidav, Analytic properties of the inverse $A^{-1}(z)$ of an analytic operator-valued function $A(z)$, Arch. Rat. Mech. Analysis 32 (1969), S. 298 - 310.

H. L. Royden, Function algebras, Bull. Am. Math. Soc. 69 (1963), S. 281 - 298.

[10] J. D. Tamarkin, On Fredholm's integral equations, whose kernels are analytic in a parameter, Annals of Math. 28 (1927), S. 127-152. 